\title{
Identification of a novel isoform of Murr1 transcript, U2mu, which is transcribed from the portions of two closely located but oppositely oriented genes
}

\author{
Youdong Wang, Keiichiro Joh*, Tsunehiro Mukai \\ Department of Biomolecular Sciences, Saga Medical School, 5-1-1 Nabeshima, \\ Saga 849-8501, Japan
}

(Received 7 June 2002, accepted 2 September 2002)

\begin{abstract}
Here we identified a novel transcript in mouse that is transcribed from the portions of two independent genes, U2af1-rs1 and Murr1, and we designated it U2mu. The U2af1-rs1 gene is located in the intron and transcribed in the opposite direction from the Murr1 gene on the proximal region of mouse chromosome 11. The $U 2 m u$ cDNA sequence is derived from three genomic regions - an intron of the Murr1 gene, an antisense sequence of U2af1-rs1 gene, and the last exon of Murr 1 gene - in the order of 5 ' to 3 '. The $U 2 m u$ transcript of $2.8 \mathrm{~kb}$ is expressed ubiquitously in adult mice. It is transcribed biallelically, and is not imprinted, in neonatal and adult mice.
\end{abstract}

The U2af1-rs1 gene is located on the proximal region of mouse chromosome 11 and within an intron of the Murr1 gene (Nabetani et al., 1997). The U2af1-rs1 gene has no intron and is a mouse homologue of a human gene, U2AF1-RS1, which encodes a $51 \mathrm{kDa}$ protein of the U2AF family, an essential component of a mammalian splicing complex (Tronchere et al., 1997). This gene is transcribed exclusively from the paternal allele in all the adult tissues that have been analyzed so far (Hatada et al., 1995; Hatada et al., 1993; unpublished results). This monoallelic and parent-specific mode of expression is known as genomic imprinting (Solter et al., 1988). The Murr1 gene is reported not to be imprinted, that is, it is expressed biallelicaly in neonatal mice (Nabetani et al., 1997). These two genes are transcribed in the opposite directions (Fig. 1).

We have attempted to identify new genes in this genomic region because imprinted genes tend to be clustered and to affect each other's allelic expression, but U2af1-rs1 is the only imprinted gene in this genomic region so far analyzed. A BLAST search was performed using the sequence of this genomic region as a query against an expressed sequence tags (ESTs) database at the NCBI Web site, (http://www.ncbi.nlm.nih.gov/dbEST/ index.html). We found a $243 \mathrm{bp}$ mouse 5'-EST sequence (GenBank accession No. W11838) that consists of an antisense sequence ( $87 \mathrm{bp}$ ) of the U2af1-rs1 gene and a sense sequence (156 bp) of the Murr1 gene in the $5^{\prime}$ and $3^{\prime}$ portions, respectively. We purchased the EST clone (Clone ID; 330049, ATCC No. 587162) from American Type Cul-

Edited by Toshihiko Shiroishi

* Corresponding author. E-mail: joh@post.saga-med.ac.jp ture Collection and sequenced the cDNA insert of 675 bp. The comparison of the sequence with the sequences of U2af1-rs1 and Murr1 cDNAs (accession Nos. D17407 and D85430, respectively) revealed that the 5 ' sequence $(87 \mathrm{bp})$ and the 3 ' sequence $(588 \mathrm{bp}$ ) were identical with the antisense sequence of U2af1-rs $1 \mathrm{cDNA}$ and the sense sequence of 3' end of Murr1 cDNA, respectively (Fig. 1). Therefore, we designated the transcript corresponding to this cDNA $U 2 m u$.

A mouse adult liver cDNA library (Life Technologies Co.) was further screened for U2mu cDNA clones using two different probes. One (probe MU) was derived from Murr1 cDNA; the other (probe U2) was derived from U2af1-rs1 cDNA. Only one cDNA clone was found to be positive for both probes in the screening of $8 \times 10^{6}$ clones. Sequencing of this cDNA clone revealed that the cDNA insert is 1459 bp long, excluding the polyA tail, (accession No. AB076722) and consists of sequences derived from three portions of the genome, that is, the intron of the Murr1 gene ( $679 \mathrm{bp}$ ), the antisense sequence of the U2af1-rs1 gene (204 bp); and the last exon of the Murr1 gene (576 bp) (Figs. 1, 2). The largest ORF in the sequence encodes a putative protein of 243 amino acids and shares its $3^{\prime}$ half portion (encoding 114 amino acids) with the ORF of Murr1 mRNA (Fig. 2). No sequence homologous to the protein was found by the BLAST search against the protein databases. The existence of U2mu mRNA was confirmed by RT-PCR of mouse adult liver RNAs with the primer pairs unk-189/UMU-SR and UMUF/UMUR (Fig. 1) (data not shown).

Using Northern blotting, we assessed the full length and the tissue distribution of $U 2 m u$ mRNA as follows: a premade Mouse Multiple Tissue Northern (MTNR) Blot 


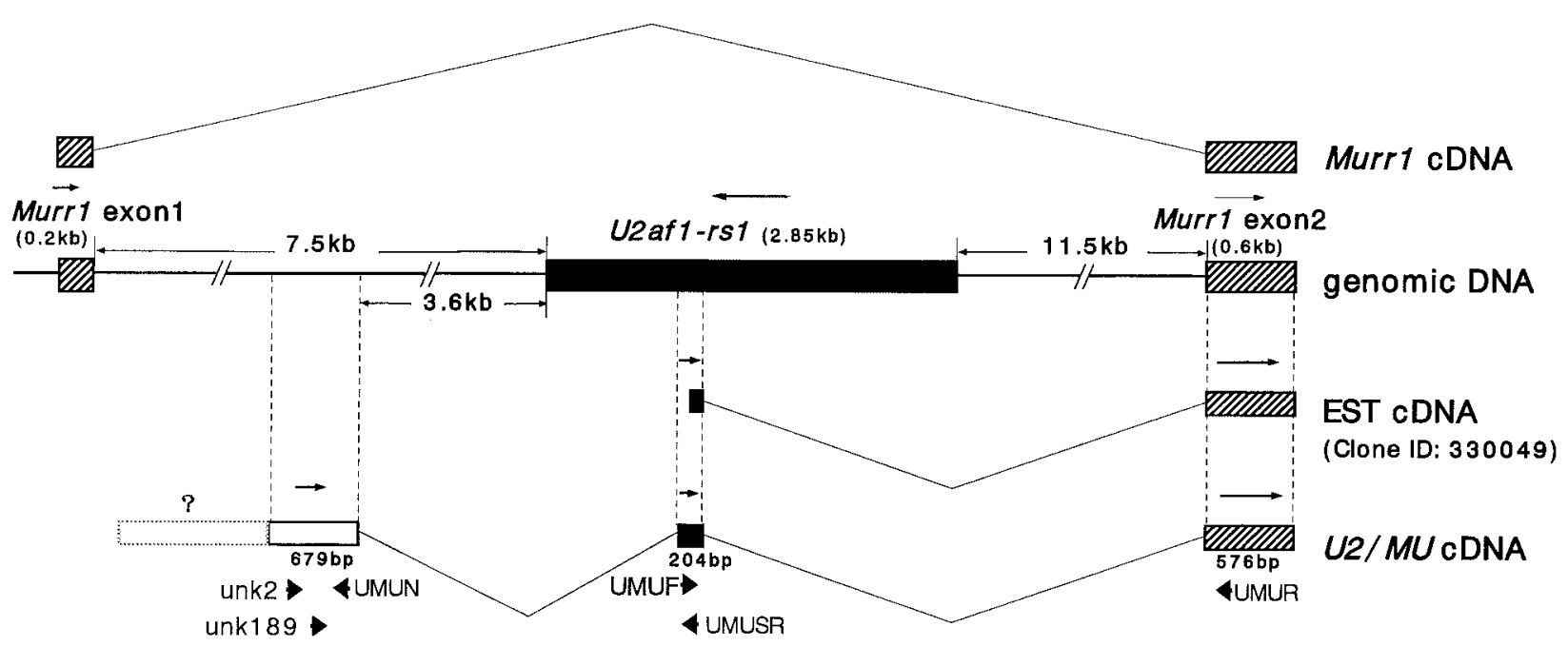

Fig. 1. Genomic structure of Murr1 and U2af1-rs1 genes. The U2af1-rs1 gene and the exons of the Murr1 gene are shown as boxes on the genomic DNA along with the Murr1 and U2mu cDNAs. Murr1 and U2mu are transcribed rightward, and U2af1-rs1 leftward. Primers used for PCR (see text) are indicated by arrowheads above and below the cDNAs.

(Clontech Co.) was probed with the PCR fragment synthesized with primers unk2 and UMUN (Fig. 1). The probe recognized RNAs of approximately $2.8 \mathrm{~kb}$ in all the tissues (Fig. 3a). This result indicates that the U2mu transcript was expressed almost ubiquitously, although at a very low level. The main tissues expressing this transcript were brain, lung, liver, and kidney. When the same blot was reprobed with the Murr $1 \mathrm{cDNA}$ probe, $1 \mathrm{~kb}$ RNAs were detected at high levels in all the tissues examined with a somewhat different distribution from $U 2 m u$ transcripts. Murr1 was expressed abundantly in heart, brain, liver, kidney, and testis (Fig. 3b).

The allelic expression of the $U 2 m u$ transcript was analyzed because its antisense gene, U2af1-rs1, is imprinted in all the tissues so far examined (Hatada et al., 1995; Hatada et al., 1993; Wang et al., unpublished data). We used a polymorphism in the $B s p H I$ site between the two mouse strains C57BL/6 (B6) and PWK at position \#1031 in the cDNA sequence (Fig. 2). PolyA RNAs were prepared from various tissues of adult $\mathrm{F} 1$ progeny obtained by reciprocal crosses between B6 and PWK. RT-PCR with primers UMUF and UMUR2 (Fig. 2) yielded a $531 \mathrm{bp}$ fragment that was suggested to $B s p H I$ digestion. Both B6 and PWK alleles were found to be expressed in all the tissues examined (Fig. 4). Biallelic expression was also observed in neonatal mice (data not shown). This biallelic expression of $U 2 m u$ transcript suggests that this transcript may not be involved in paternal allele-specific expression of the U2af1-rs1 gene because antisense transcripts, which have been reported to be involved in the imprinting of their counterpart genes, show reciprocal monoallelic expression (Lyle et al., 2000; Chamberlain et al., 2001).

We hypothesize that the mouse U2af1-rs1 gene was formed by retro-transposition of its ancestral gene on the
$\mathrm{X}$ chromosome into the intron of the ancestral Murr1 gene in mice arose after mice diverged from humans, because the gene is an intron-less gene and its human homolog has not been identified in the intron of the human MURR1 gene (Nabetani et al., 1997). This fact indicates that the $U 2 m u$ transcript must have been formed after the divergence between mice and humans via the combination of two sequences, the newly formed sequence, U2af1-rs1 and the old sequence, Murr1. A full-length cDNA clone could not be isolated in spite of extensive screening of the mouse cDNA library, probably due to the very low level of expression of the gene. 5' RACE was also performed to obtain the sequence of the 5' part of the mRNA but the attempt failed, again due to the low level of expression and also due to the large size of the missing portion of the mRNA. We do not know, therefore, the location of the promoter region of the gene. One can consider that the $U 2 m u$ transcript is a splicing variant of the Murr1 transcript that is formed due to the new splicing sites created by the transposition of the U2af1-rs1 sequence into the intron of the Murr1 gene, although it is also possible that the $U 2 m u$ transcript is possible to be transcribed from a different promoter. We do not know which possibility is correct, but the former case seems more likely at present.

We do not know whether the $U 2 m u$ transcript has any functions in mice. U2mu mRNA has an ORF that starts in the sequence from the Murr1 intron, continues to the sequence from U2af1-rs 1 and is connected to the sequence from the Murr1 exon that is shared by the Murr1 ORF (Fig. 2). We have not identified the protein coded by the ORF yet, but the ORF may be functional because a long sequence $(342 \mathrm{bp})$ is connected in-frame to the 3 ' half of the Murr1 ORF without interruption by stop codons. The ORF in the $U 2 m u$ transcript suggests that the transcript 


\begin{tabular}{|c|c|}
\hline CTCACAGGATCCACTGCGTS GTCTCPAAGTEC & \\
\hline ITGGTPTGGGGGGAGTCA AGT SCAGATGGT CAGGCTTSGCAGCAGTGTCCTTISCTGCT & 120 \\
\hline GCCATCTCATH AGCSCAPCCATCTTA ATAAA A GGGAACATACATAGATATAT CATGT & 180 \\
\hline 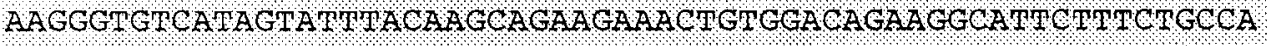 & 240 \\
\hline 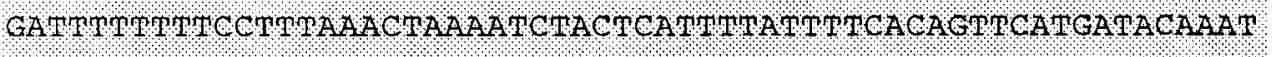 & 300 \\
\hline GAAACTACTT TAA A A A A A A STCTCTCTACTCSTAGCAAAA ACTSTICCCTACGCTAGATA & \\
\hline TGGGGAGAACT T GCTTGTCATACCACTATA A A ATACTCCGGT GTAGCECCATGGTCTGA & \\
\hline 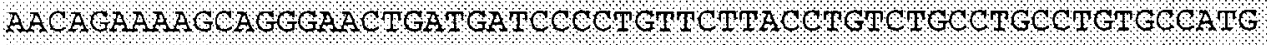 & 48 \\
\hline CAATGCCAGGLCAGTGCCATCTCATCACTGGAGGCCTGGACACCAAGGGGATGTGTCTCT & 540 \\
\hline 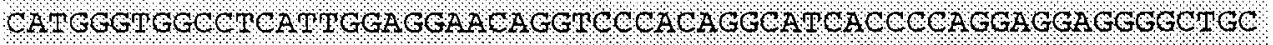 & 600 \\
\hline I & \\
\hline 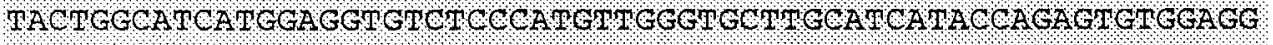 & 660 \\
\hline 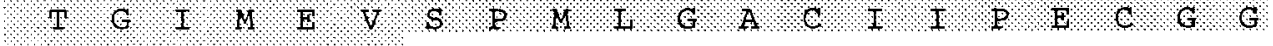 & \\
\hline AৎCCCGGGA CTGGAGCCAGAATTCACACTGGAGCTGTCGTCCTGCGTACCATCTTCCAT & \\
\hline 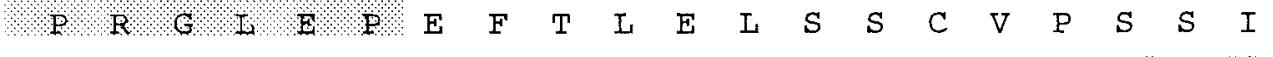 & \\
\hline AAAGAGAGAGAGGGCTGCTTGGCATTCTTCTTCCGACTGGTACTGAACATACACATTGC & \\
\hline $\begin{array}{llllllllllllllllllll}\mathrm{K} & \mathrm{E} & \mathrm{R} & \mathrm{E} & \mathrm{G} & \mathrm{C} & \mathrm{L} & \mathrm{A} & \mathrm{F} & \mathrm{F} & \mathrm{F} & \mathrm{R} & \mathrm{L} & \mathrm{V} & \mathrm{L} & \mathrm{N} & \mathrm{I} & \mathrm{H} & \mathrm{I} & \mathrm{A}\end{array}$ & \\
\hline CCCGCAGATGAGGTTCCAGGTTGCAGCTTACTTTGAACTGAATCACCTTTCCCACGTTCT & \\
\hline $\begin{array}{lllllllllllllllllllll}\mathrm{P} & \mathrm{Q} & \mathrm{M} & \mathrm{R} & \mathrm{F} & \mathrm{Q} & \mathrm{V} & \mathrm{A} & \mathrm{A} & \mathrm{Y} & \mathrm{F} & \mathrm{E} & \mathrm{L} & \mathrm{N} & \mathrm{H} & \mathrm{L} & \mathrm{S} & \mathrm{H} & \mathrm{V} & \mathrm{L}\end{array}$ & \\
\hline GAACTCCGGCAGCACGTCATGGTAGAAATCCAAGAACTGCTGTCTATTGCATCTGCAGA & \\
\hline $\begin{array}{llllllllllllllllllll}\mathrm{E} & \mathrm{L} & \mathrm{R} & \mathrm{Q} & \mathrm{H} & \mathrm{V} & \mathrm{M} & \mathrm{V} & \mathrm{E} & \mathrm{I} & \mathrm{Q} & \mathrm{E} & \mathrm{L} & \mathrm{L} & \mathrm{S} & \mathrm{I} & \mathrm{A} & \mathrm{S} & \mathrm{A} & \mathrm{D}\end{array}$ & \\
\hline САTGGATTTCAACCAGTTAGAGGCATTCCTGACTGCTCAAACCAAAAAGCAAGGTGGCAT & \\
\hline $\begin{array}{llllllllllllllllllll}M & D & F & N & Q & L & E & A & F & L & T & A & Q & T & K & K & Q & G & G & I\end{array}$ & \\
\hline CACCAGTGAGCAAGCTGCAGTCATCTCCAAGTTTTGGAAGAGCCACAAGATAAAAATCCG & 1020 \\
\hline 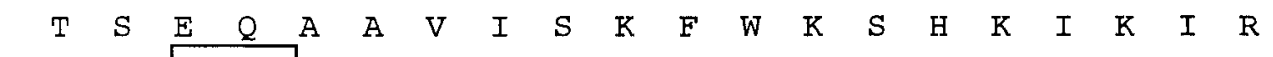 & \\
\hline AGAGAGTdTCATGAAGCAGAGCCGCTGGGACAACGGCCTTCGGGGCCTGAGCTGGAGAGT & 108 \\
\hline $\begin{array}{llllllllllllllllllll}E & S & L & M & K & Q & S & R & W & D & N & G & L & R & G & L & S & W & R & V\end{array}$ & \\
\hline CGATGGCAAGTCTCAGTCACGGCACTCAACTCAGATACACAGCCCTGTTGCCATAATAGA & 11 \\
\hline $\begin{array}{llllllllllllllllllll}D & G & K & S & Q & S & R & H & S & T & Q & I & H & S & P & V & A & I & I & E\end{array}$ & \\
\hline GCTGGAATTTGGAAAAAATGGACAGGAATCTGAATTTTTGTGTCTGGAATTTGATGAAGT & 1200 \\
\hline $\begin{array}{llllllllllllllllllll}L & E & F & G & K & N & G & Q & E & S & E & F & L & C & L & E & F & D & E & V\end{array}$ & \\
\hline TAAAGTCAAGCAAATCCTGAAGAAGCTGTCAGAGGTAGAAGAGAGTATCAACAGGCTGAT & 1260 \\
\hline $\begin{array}{llllllllllllllllllll}K & V & K & Q & I & L & K & K & L & S & E & V & E & E & S & I & N & R & L & M\end{array}$ & \\
\hline $\begin{array}{l}\text { GCAGGCAGCCTAACTGAAGAGAGTATCAATAGGCTGATGCAGGCAGCCTAACTGAAGGCT } \\
\text { Q A A * }\end{array}$ & 1320 \\
\hline GGAGGAAGGGGCGTTTGAAGTGAAGCTGCTCACAGACTTTCTCCACTGAC & 138 \\
\hline TCCTGTTTGCCCACTGGTGTTACCAAAAGACATTGTATACATGCATGAAAGTC & 14 \\
\hline TAAATAAAAATATATTTT-poly-A & \\
\hline
\end{tabular}

Fig. 2. Nucleotide sequence of $U 2 m u$ cDNA and the deduced amino acid sequence. The shaded sequence from \#1 to \#679 comes from the intron of the Murr1 gene. The underlined one from \#680 to \#883 is an antisense sequence of U2af1-rs1 and the last part is from the last exon of Murr1 (see Fig. 1). The 3' portion of the ORF is identical to that of the Murr1 ORF (data not shown). The boxed sequence (\#1029-1034) is a polymorphic BspHI site used for the analysis of allelic expression of the U2mu gene. The polyA signal is underlined. The two arrows indicate the positions of the primers, UMUF and UMUR2, used for the analysis of allelic expression in Fig. 4.

has a function different from that of the Murr1 transcript.

In the evolution of eukaryotes new genes are thought to emerge mainly through two processes, that is, gene duplication followed by divergence of the duplicated genes and shuffling of exons derived from different genes by unequal crossing-over or transposition (Ohta, 2000). The
$U 2 m u$ transcript, however, does not result from either of these processes. This transcript consists of sequences from at least three exons. The second exon is transcribed from a sequence that is thought to have transposed from another location where the sequence was not transcribed in this direction. We are interested in the 


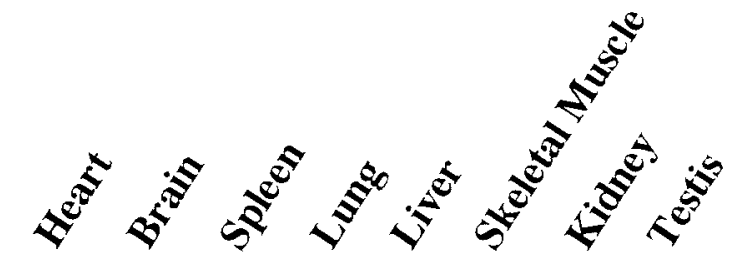

a.

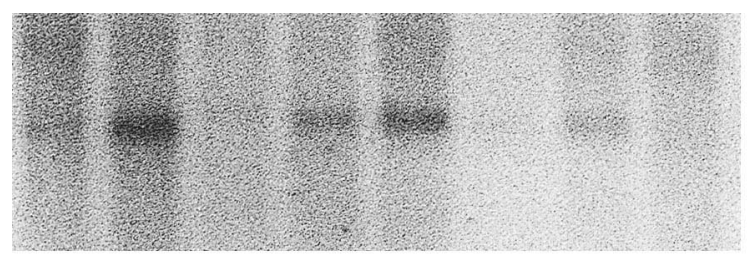

b.

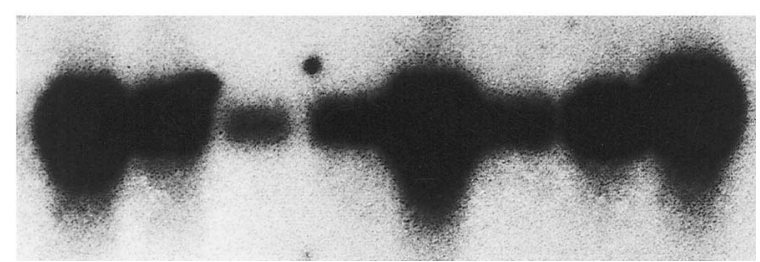

Fig. 3. Northern blot analyses of $U 2 m u$ and Murr1 in adult mouse tissues. a) Probed with $U 2 m u$ probe, exposed for 2 weeks with an intensifying screen. b) Probed with Murr1 probe, exposed for 24 hours with an intensifying screen.

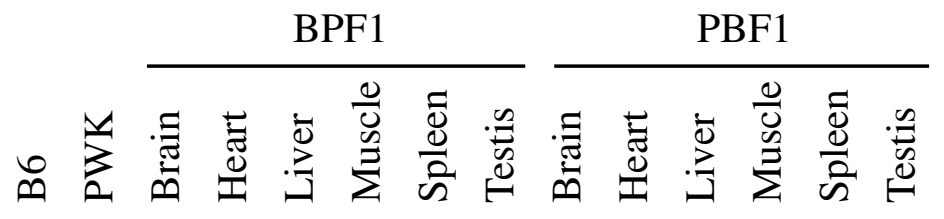

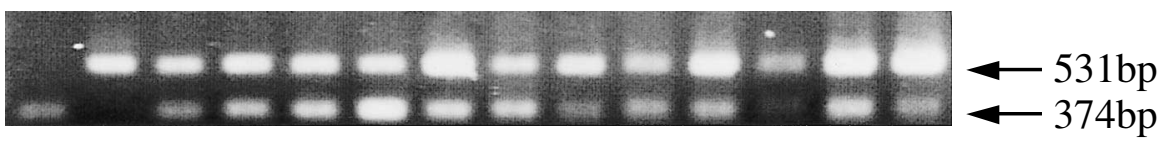

Fig. 4. Biallelic expression of $U 2 m u$ transcript. RT-PCR was performed on polyA RNAs from the tissues of adult mice. The $531 \mathrm{bp}$ cDNA products were digested with BspHI. B6 cDNA was cut with the enzyme (lane B6), whereas PWK cDNA was not (lane PWK). Both alleles were expressed in all the tissues examined of F1 hybrid mice between these strains, BPF1 (B6 female x PWK male) and PBF1 (PWK female x B6 male).

process of the formation of the $U 2 m u$ transcript (whereby the transposed sequence was connected as a new exon to preexisting sequences, the intron and an exon sequence of the Murr1 gene) because this may be a new example of the process of the emergence of a new gene. It may be useful to identify which animals have homologues of U2af1-rs1 and U2mu, and which animals do not in the phylogenetic tree. Comparison of the corresponding sequences among these animals may give important insights into the process of the emergence of the $U 2 m u$ transcript.

We thank Mr. S. Takuma at the Center for Laboratory Animals for his technical assistance. This work was supported by a Grant-in-Aid from the Ministry of Education, Culture, Sports, Science, and Technology in Japan.

\section{REFERENCES}

Chamberlain, S. J., and Brannan, C. I. (2001) The Prader-Willi syndrome imprinting center activates the paternally expressed murine Ube3a antisense transcript but represses paternal Ube3a. Genomics 73(3), 316-22.

Hatada, I., Kiragawa, K., Yamaoka, T., Wang, Z., Arai, Y., Hashido, K., Ohishi, S., Masuda, J., Ogara, J., and Mukai, T. (1995) Allele-specific methylation and expression of an imprinted U2af1-rs1 (SP2) gene. Nucleic Acids Res. 23, $36-41$.

Hatada, I., Sugama, T., and Mukai, T. (1993) A new imprinted gene cloned by a methylation-sensitive genome scanning method. Nucleic Acids Res. 21, 5577-5582.

Lyle, R., Watanabe, D., Vruchte, D., Lerchner, W., Smrzka, O. W., Wutz, A., Schageman, J., Hahner, L., Davies, C., and Barlow, D.P. (2000) The imprinted antisense RNA at the Igf2r locus overlaps but does not imprint Mas1. Nature 
Genetics 25, 19-21.

Moore, T., Constancia, M., Xubair, M., Bailleul, B., Feil, R., Sasaki, H., and Reik, W. (1997) Multiple imprinted sense and antisense transcripts, differential methylation and tandem repeats in a putative imprinting control region upstream of mouse Igf2. Proc. Natl. Acad. Sci. USA 94, 12509-12514.

Nabetani, A., Hatada, I., Morisaki, H., Oshimura, M., and Mukai, T. (1997) Mouse U2af1-rs1 is a neomorphic imprinted gene. Mol. Cell. Biol. 17, 789-798.

Ohta, T. (2000) Evolution of gene families. Gene 259(1-2), 4552.

Rougeulle, C., Cardoso, C., Fontes, M., Colleaux, L., and Lalande, M. (1998) An imprinted antisense RNA overlaps
$U B E 3 A$ and a second maternally expressed transcript. Nature Genetics 19, 15-16.

Solter, D. (1988) Differential imprinting and expression of maternal and paternal genomes, Annu. Rev. Genet. 22, 127-146.

Tronchere, H., Wang, J., and Fu, X. D. (1997) A protein related to splicing factor U2AF35 that interacts with U2AF65 and SR proteins in splicing of pre-mRNA. Nature 388, 397400.

Wutz, A., Smrzka, O. W., Schweifer, N., Schellander, K., Wagner, E. F., and Barlow, D. P. (1997) Imprinted expression of the Igf2 $r$ gene depends on an intronic CpG island. Nature 389, 745-749. 